\title{
Phase I/II Study of Gefitinib and Concomitant Preoperative Radiotherapy in Patients with Locally Advanced Rectal Cancer
}

\author{
Abdelkarim S. Allal ${ }^{1,2 \#}$, Arnaud D. Roth ${ }^{3}$, Alessandra Franzetti-Pellanda ${ }^{4}$, Marta Bonet ${ }^{2,5}$, \\ Pascal Gervaz ${ }^{3}$, Sabine Bieri ${ }^{6,7}$ \\ ${ }^{1}$ Department of Radiation Oncology, HFR-Hôpital Cantonal de Fribourg, Fribourg, Switzerland; ${ }^{2}$ Department of Radiation Oncology, \\ University Hospital of Geneva, Geneva, Switzerland; ${ }^{3}$ Department of Surgery, University Hospital of Geneva, Geneva, Switzerland; \\ ${ }^{4}$ Oncologic Institute of Southern Switzerland, Hospital San Giovanni, Bellinzona, Switzerland; ${ }^{5}$ Radiation Oncology Center, Con- \\ sorci Sanitari de Terrassa, Institut Oncològic del Vallès (CST-HGC-CSPT), Terrassa-Barcelona, Spain; ${ }^{6}$ Radiation Oncology Center, \\ Hospital of Sion, Sion, Switzerland; ${ }^{7}$ Radioonkologie Amsler AG, Liestal, Switzerland. \\ Email: \#allala@h-fr.ch
}

Received September $19^{\text {th }}, 2012$; revised October $18^{\text {th }}, 2012$; accepted October $29^{\text {th }}, 2012$

\begin{abstract}
Objective: To determine the recommended dose (RD) of gefitinib when combined with concomitant radiotherapy (RT) in a preoperative setting in patients with locally advanced rectal cancer. Secondary objectives were to evaluate acute toxicities, pathological response rate, progression-free and overall survival (OS). Materials and Methods: 20 patients with cT3-4 or $\mathrm{cN}+\mathrm{cM} 0$ tumors were enrolled. The planned RT consisted in 50 Gy given in 2 daily fractions of $1.25 \mathrm{~Gy}$ in 4 weeks. During RT, gefitinib was planned to be given orally once daily with 2 successive dose levels: $250 \mathrm{mg}$ and $500 \mathrm{mg}$. Rectal surgery was scheduled $5-6$ weeks after completion of RT. The median follow-up for all patients was 57 months. Results: Among the first cohort of 6 patients, 1 patient presented a dose limiting toxicity (DLT) (Grade 3 diarrhea/dehydration). In the second cohort, 2/6 patients presented with the same DLT so that $250 \mathrm{mg}$ was considered as the RD. Main acute toxicities consisted in diarrhea (grade $2-3,63 \%$ ), and skin reaction (in RT fields grade $2-3$ in $42 \%$ ). The 5-year actuarial OS and loco-regional control rates were of $80 \%$ and $84 \%$ respectively. Conclusion: The concomitant daily administration of $250 \mathrm{mg}$ of gefitinib with 50 Gy preoperative RT is feasible with manageable toxicity. The major pathologic response rate is encouraging, though it needs further confirmation. Distant metastasis still represents a concern and new strategies to overcome this issue are warranted.
\end{abstract}

Keywords: Rectal Cancers; Radiotherapy; Gefitinib; Acute Toxicity

\section{Introduction}

Preoperative RT with or without chemotherapy (CT) is now considered a standard therapy in locally advanced rectal cancer [1]. Indeed, even in case of optimal surgery with a total mesorectal excision, preoperative $\mathrm{RT}$ reduces local recurrences and may improve survival [2-4]. Strategies aiming at improving the efficacy of the neo-adjuvant treatment consisted either in RT fractionation alteration and/or the addition of new chemo- or targeted therapies $[5,6]$. We have conducted a phase I-II study [7] and concluded that, in locally-advanced rectal cancers, preoperative hyper fractionated RT (HFRT) to a total dose of 50 Gy was feasible, with acceptable acute and late toxicity and objective down staging effect. The toxicity profile

${ }^{*}$ This study was sponsored by Astra-Zeneca Company.

\#Corresponding author. was such a room was left for additional intensification of the treatment by adding new chemotherapeutic or biological agent, hence the present study.

Gefitinib is a potent and selective inhibitor of the epidermal growth factor (EGF). It's a drug directed toward the intracellular tyrosine kinase domain of EGFR which showed an increase growth inhibition in cancer cells and also an enhancement of ability of radiation to directly kill the stem cancer cells through alteration of signal transduction, inhibition of DNA damage repair and improvement of re-oxygenation during fractionated RT [8]. There is now considerable evidence documenting the expression and over-expression of EGFR in an extensive range of human cancers (lung, breast, head and neck, prostate, and colorectal). In head and neck cancers EGFR inhibitor added to RT have demonstrated a significant improvement in local control and survival compared with RT 
alone [9]. Williams et al. [10] studied the antitumor efficacy of combined irradiation and gefitinib in a human colon cancer xenograft and showed an enhanced radiosensitivity of the xenografts to both single-dose and fractionated RT.

Thus the phase I part of the present study was to evaluate the tolerance of RT when combined with gefitinib in a neoadjuvant setting in locally advanced rectal cancers. The recommended dose (RD) of gefitinib was to be determined. The phase II part of the study intended to investigate the efficacy of the combined regimen as measured by the rate of complete pathological tumour response, the rate of clear margin resection (particularly in T4 tumours), and the classical oncologic endpoints.

\section{Material and Methods}

\subsection{Eligibility Criteria}

This study was performed after approval by a local institutional Ethic Committee and informed consent was obtained from each patient. Twenty patients from three Swiss institutions (Geneva University Hospital, San Giovanni Hospital Bellinzona and Sion Hospital) were included in this prospective trial. Eligibility criteria included the following : histologically-confirmed operable adenocarcinoma of the rectum; clinical stage T3-4, N0-2 or T1-2, N1-2, M0; World Health Organization performance status of $0-2$; age between $18-75$ years; no prior (in the last 5 years) or concurrent malignancy (except non-melanoma skin cancers or in-situ carcinoma of the cervix) ; no prior RT or CT. Patients were excluded if they were considered inoperable for reasons of disease extension or general condition. Known severe hypersensitivity to gefitinib or any recipients of this product, clinically active interstitial lung disease, pregnancy or breast feeding, concomitant use of phenytoin, carbamazepine, rifampicin, barbiturates, evidence of any other significant clinical disorder or laboratory finding that makes the subject undesirable to participate in the study, haemoglobin $<10 \mathrm{~g} / \mathrm{dl}$, WBC $<3500 / \mathrm{ml}$, platelets $<$ $100,000 / \mathrm{ml}$, serum bilirubin $>$ than 1.25 times the upper limit of the reference range, estimated creatinine clearance (Cockcroft) $<40 \mathrm{ml} / \mathrm{min}$ were exclusion criteria's as well.

\subsection{Pre-Treatment Evaluation}

All patients underwent before study treatment physical examination including digital rectal examination, proctoscopy and/or colonoscopy, abdomino-pelvic computed tomography (CT), endorectal ultrasound (EUS) (when available) or pelvic magnetic resonance imaging (MRI), chest x-ray, and complete blood chemistry and tumour marker (blood count, renal and liver functions, CEA). All patients had an electrocardiogram. Disease was staged using EUS and/or MRI and rectal examination.

\subsection{Treatment, Tumour Response and Toxicity Evaluation}

The RT schedule was the same as published previously [5]. High energy photon beams of at least $6 \mathrm{MV}$ have to be used with a 3-field technique (one posterior and two lateral fields). The total dose of 50 Gy in 40 fractions of $1.25 \mathrm{~Gy}, 2$ times per day, with at least 6 hours' interval (45 Gy to the posterior pelvic volume and 5 Gy boost to the known macroscopic disease) has to be given. For three-dimensional treatment planning purposes, all patients should have a computed tomography scanning in the treatment position.

The gefitinib dose levels for this study were 250 (one tablet) and $500 \mathrm{mg}$ (two tablets) daily (5 days a week) starting on day 1 of RT. It was planned to enroll cohorts of 6 subjects in a step-wise fashion. For each step, if no subject experiences a dose-limiting toxicity (DLT) within 6 weeks of follow-up, the dose was to be escalated for the next cohort of six subjects. Where one subject out of a cohort of six experiences a DLT, the cohort will be expanded to 12 subjects. If an additional DLT occurs in the six added subjects, the MTD is considered to be reached and this will be considered as the recommended dose (RD). A final cohort of $6-8$ patients was to be treated at the RD as part of the phase II of the study.

Acute toxicities were to be defined as all toxicities occurring before surgery. DLT is defined as any grade 4 reaction, Grade 2 hepatic or renal toxicity (except reversible grade 3 liver transaminase elevation), Grade 3 diarrhea despite aggressive anti-diarrheal therapy, other Grade 3 non-hematological toxicity (excluding alopecia, nausea, vomiting or skin), and treatment delay $>2$ weeks due to toxicity. Acute toxicities were to be graded using the National Cancer Institute common toxicity criteria V.2.0 [11], and late complications using the Radiation Therapy Oncology Group grading system (RTOG).

\subsection{Surgery, Histopathologic Assessment and Adjuvant Treatment}

Surgery was to be performed within 6 weeks of the completion of treatment. Total mesorectal excision (TME) was the recommended surgical technique. The extent and type of the operative procedure was left to the discretion of the surgeon.

Tumour response was evaluated according to the following pathological criteria:

1) Pathological complete response (pCR): total absence of any tumour tissue. 
2) Pathologic partial response (pPR): persistence of a few islands of microscopic tumour cells having a maximum dimension of about $10 \mathrm{~mm}$.

3) Minor or no response $(\mathrm{mnR})$ : any response with more than residual disease as defined above.

In case of complete tumour regression or of minimal residual disease, a complete inclusion of the potential tumour site was to be performed with sections of 2 - 3 $\mathrm{mm}$, while for macroscopic residual disease sections of 5 - $10 \mathrm{~mm}$ were to be performed. Clinical and pathological staging was assessed according to the sixth edition (2002) of Union International Contre le Cancer classification.

In cases of $\mathrm{T} 4$ or $\mathrm{N}+$ disease, adjuvant $\mathrm{CT}$ had to be administered after surgery according to local policy.

\subsection{Statistics}

Rates of acute and late toxicities as well as rates of pathologic response were calculated as simple proportions. Actuarial local control, DFS and OS rates were calculated by the product-limit method (Kaplan-Meier method). All analysis was performed using the Statview V5 software (SAS Institute Inc.).

\section{Results}

\subsection{Patient's Characteristics}

Twenty patients were included into the study between October 2003 and April 2006. Patients and tumour characteristics are listed in Table 1. The median distance from the anal margin to the lower edge of the tumour was $4.5 \mathrm{~cm}$ (range, $1-11 \mathrm{~cm}$ ).

Table 1. Patients characteristics $(n=20)$.

\begin{tabular}{ccc}
\hline & $\mathrm{n}$ & $\%$ \\
\hline $\begin{array}{c}\text { Gender, male/female } \\
\text { WHO performance status } 0 / 1\end{array}$ & $19 / 1$ & $15 / 5$ \\
Age, median (range) & $58.5(35-72)$ years \\
Clinical TNM stage (UICC 2002) & \multicolumn{2}{c}{} \\
T2 & 3 & 15 \\
T3 & 16 & 80 \\
T4 & 1 & 5 \\
N0 & 7 & 35 \\
N1 & 7 & 35 \\
N2 & 5 & 25 \\
Nx & 1 & 5 \\
Histology Grading & & \\
G1 & 4 & 20 \\
G2 & 11 & 55 \\
G3 & 3 & 15 \\
Gx & 2 & 10 \\
\hline
\end{tabular}

\subsection{Gefitinib Administration and Dose Escalation}

The occurrence of a dose-limiting toxicity in the first treated patients with Grade 3 diarrhea, dehydration, hospitalization required withdrawal of gefitinib treatment. After the 6 first treated patients, and as per protocol, a cohort of 6 other patients was treated at the first dose level of $250 \mathrm{mg} /$ day. Among the later cohort two DLT of the same acute intestinal toxicity occurred giving a rate of DLTs of $25 \%(3 / 12)$. Thus the dose escalating schedule was abandoned and the dose of $250 \mathrm{mg}$ /day of gefitinib was considered as the RD. No other DLT occurred in the last cohort of 8 patients treated with the RD. In total gefitinib was stopped definitively in two patients (2nd and 4th week) due to acute toxicities.

\subsection{Treatment Compliance and Acute Toxicities}

All patients completed the RT schedule, receiving 45 Gy to the pelvis and $50 \mathrm{~Gy}$ to the rectal tumour, using 3 fields technique with X15-18 MV photon beams. RT was temporarily interrupted in 3 patients for duration of 1 day. In all patients the interruption was due to acute toxicities. The median RT duration was 28 days (range: 26 - 30).

The acute toxicities related to the combined treatment are detailed in Table 2. All were reversible after conservative interventions. There were no grade 4 events and no grade $2-4$ hematologic or infectious events. The Grade 3 acute toxicities concerned mainly the skin and the gut. Three patients presented with grade 3 intestinal complications requiring hospitalization in two cases. Four patients presented with grade 3 skin reactions, all had tumour located at the lower part of the rectum $(<6$ $\mathrm{cm}$ of the anal margin). Those patients were treated with the anal margin included in the RT fields.

\subsection{Surgery, per Operative Complications and Tumor Response}

Surgery was performed at a median interval of 42 days (range, 26 - 55 days) after the end of the neo-adjuvant therapy. Surgical procedures included abdomino-perineal resection (APR) in 10 patients $(50 \%)$ and low anterior resection (LAR) in 10 patients $(50 \%)$. Except one, all patients were resected with tumour-free margins. There were no immediate per operative complications. One patient developed anastomotic leakage and perineal abscess occurred in 2 patients 8 weeks after surgery.

The pathologic response information and $\mathrm{TN}$ down staging are listed in Table 3. Complete pathologic response was observed in 4 patients $(20 \%)$ and major response in 6 patients $(30 \%)$. 
Table 2. Acute toxicities according to the National Cancer Institute common toxicity criteria V.2.0 for all patients $(\mathrm{n}=$ 20).

\begin{tabular}{cccccc}
\hline & Grade & & & & \\
\hline Organ/Symptom & $\mathbf{0}$ & $\mathbf{1}$ & $\mathbf{2}$ & $\mathbf{3}$ & $\mathbf{4}$ \\
\hline Hemoglobin & 13 & 3 & 0 & 0 & 0 \\
Leucocytes & 12 & 4 & 0 & 0 & 0 \\
Thrombocytes & 16 & 0 & 0 & 0 & 0 \\
Skin $^{*}$ & 5 & 7 & 4 & 4 & 0 \\
Infection & 16 & 1 & 0 & 0 & 0 \\
Diarrhea & 3 & 5 & 9 & 3 & 0 \\
Abdominal pain & 17 & 3 & 0 & 0 & 0 \\
Nausea/vomiting & 16 & 2 & 1 & 1 & 0 \\
Proctitis/tenesmus & 11 & 8 & 1 & 0 & 0 \\
Urogenital & 19 & 1 & 0 & 0 & 0 \\
Fatigue & 9 & 9 & 2 & 0 & 0 \\
Pharyngitis & 19 & 1 & 0 & 0 & 0 \\
\hline
\end{tabular}

*6 patients presented with acne on thorax or face.

Table 3. Pathologic stage (ypT) compared with clinical stage (cT) at baseline $(n=20)$.

\begin{tabular}{ccccc}
\hline \multicolumn{5}{c}{ Pathologic stage } \\
\hline Clinical T stage & ypT0 & ypT1 & ypT2 & ypT3 \\
\hline cT2 $(\mathrm{n}=3)$ & 1 & 0 & 2 & 0 \\
cT3 $(\mathrm{n}=16)$ & 3 & 2 & 6 & 4 \\
cT4 $(\mathrm{n}=1)$ & 0 & 0 & 0 & 2 \\
Total (n=20) & 4 & 2 & 8 & 6 \\
Clinical N stage & ypN0 & ypN1 & ypN2 & \\
cNo (n=7) & 6 & 0 & 1 & \\
cN1 (n=7) & 5 & 1 & 1 & \\
cN2 (n=5) & 4 & 1 & 0 & \\
cNx (n=1) & 1 & 0 & 0 & \\
Total (n=20) & 16 & 2 & 2 & \\
Tumor response & & & & \\
pCR & $4(20 \%)$ & & & \\
pPR & 6 & & & \\
mnR & 10 & &
\end{tabular}

pCR: Pathological complete response; pPR: Pathologic partial response; $\mathrm{mnR}$ : Minor or no response.

\subsection{Postoperative Adjuvant Chemotherapy}

The choice of adjuvant CT was individualized on the basis of initial tumour stage, performance status $+/-$ comorbidities, and the postoperative histopathological findings. Of the 13 patients who received adjuvant $\mathrm{CT}$, a 5 FU based CT was administrated in 8 patients (including Folfox in 2) and Xeloda based CT in 5 patients.

\subsection{Tumour Control and Survival}

The median follow-up time was of 57 months (range: 6 78 months). At last Follow-up, 15 patients were alive and
5 deceased, all from cancer. Three patients presented with loco-regional recurrences (including the one with $\mathrm{R} 1$ resection) and one patient was salvaged. Six patients developed distant metastases either as first or second event (including one with liver metastasis discovered at time of surgery). The 5-year actuarial overall and disease-free survival rates were of $80 \%$ and $55 \%$ respectively (Figure 1). The 5-year actuarial loco-regional control rate was of $84 \%$ (Figure 2).

\section{Discussion}

In the recent years, the increasing knowledge of the molecular biology of cancers allowed the identification of several biological factors responsible for the aggressiveness of tumours and this permitted the development of targeted therapies against those specific factors. EGF was one of the most studied factors and its inhibitors have been extensively explored particularly in cancers that express or over express the EGFR as H\&N and lung cancers

[http://medicalgenome.kribb.re.kr/GENT/analysis-1.php ]. In rectal cancer experiences with EGR inhibitors are scare and their tolerability and efficacy when associated with RT has not been demonstrated formally.

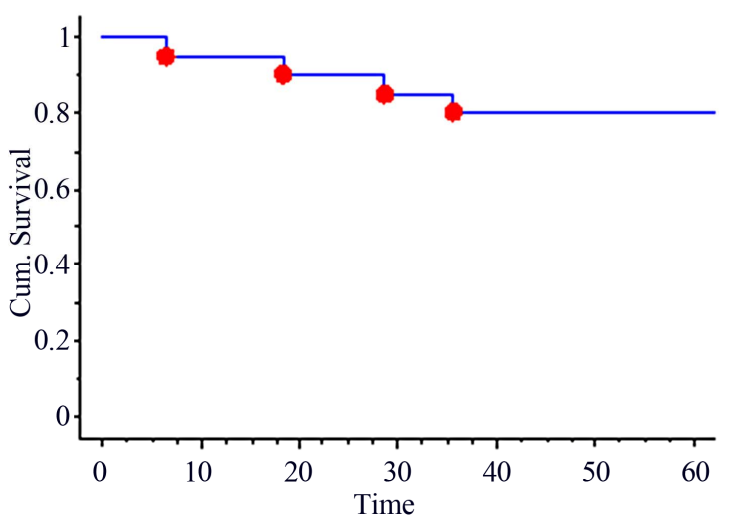

Figure 1. 5-year actuarial overall survival.

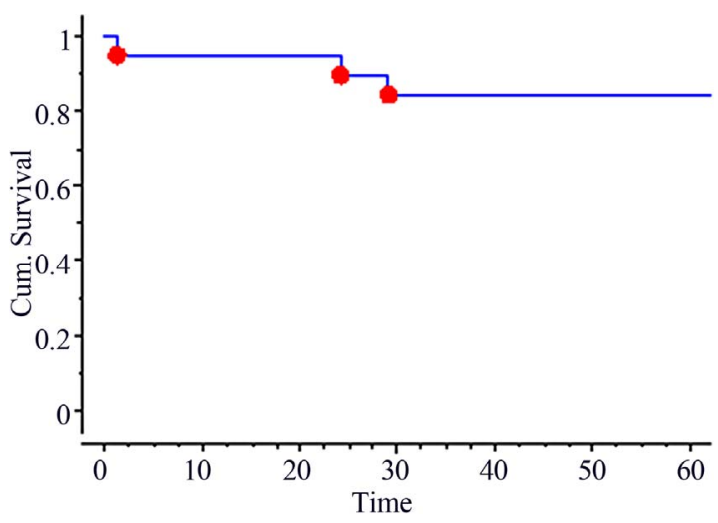

Figure 2. 5-year actuarial loco-regional control. 
The main aim of the present study was to determine the RD of gefitinib, when combined concomitantly with hyper fractionated RT in a preoperative setting in rectal cancer patients. While in the phase I part of the study it was planned a dose escalation from $250 \mathrm{mg}$ to $500 \mathrm{mg}$ daily of gefitinib, the occurrence of a DLT in one patient in the first cohort of 6 patients and than 2 DLT's in the second cohort of 6 patients, prompted us to abandon the escalation project as per protocol specifications and we considered the $250 \mathrm{mg}$ as the RD. The third cohort of 8 patients took part in the phase II part of the study and to confirm the RD. Indeed no other DLT occurred in this later cohort. Thus all patients received a daily oral dose of $250 \mathrm{mg}$ gefitinib during radiotherapy. In our study the DLT's concerned exclusively the intestinal tract with diarrhea and abdominal pain as the main symptoms. The second main side effect concerned the skin either in RT field with grade 3 toxicities observed in 4 patients $(20 \%)$ or out RT field with the well known side effect of the EGF inhibitors namely "acne rush" (6 patients 30\%). Those finding are in accordance with the finding of the other studies that used gefitinib either alone or concomitantly with chemotherapy and RT. Thus, in a phase I-II study Valentini et al. [6] reported a rate of Grade 3 toxicity of $41 \%$ when using daily $500 \mathrm{mg}$ of gefitinib and 5-FU-based chemotherapy concomitantly with RT. The most frequent toxicity was gastrointestinal with diarrhea in the majority of the cases $(20.5 \%)$, followed by skin toxicity (15.3\%). While the authors conclude that $500 \mathrm{mg}$ daily of gefitinib can be administered without any lifethreatening toxicity, due to the high rate of gastro-intestinal toxicities they suggest limiting the dose to $250 \mathrm{mg}$. This seems to us to be a wise conclusion which is in line with ours, particularly when taking into account the fact that a dose reduction of gefitinib was necessary in 24 patients $(61.5 \%)$ in their study.

RT, 5-FU-based (or analogues) chemotherapy and now EGFR inhibitors are well known toxic agents for the intestinal tract and their combination should be done with caution. Indeed in a phase I-II study, Czito et al. [5] reported an increase of intestinal toxicities when combining gefitinib $250 \mathrm{mg}$ daily, capecitabine (650 - 825 $\mathrm{mg} / \mathrm{m}^{2} / \mathrm{bid}$ ) and RT in the preoperative setting of pancreatic and rectal cancer patients. Again diarrhea was the main DLT in this study and no RD of gefitinib was suggested. The same conclusions concerning these specific toxicities were reported in studies combining other EGFR inhibitors with chemotherapy and RT in rectal cancer patients [12-14].

In the present study the secondary endpoints were to evaluate the objective tumor pathological response, quality of surgical margins, and accessory loco-regional con- trol, PFS and OS.

Potential advantages of preoperative over postoperative radio-chemotherapy are lesser acute toxicity, tumor down staging potentially resulting in a higher rate of sphincter preservation for distal tumors, improved locoregional control and disease-free survival rates. In rectal cancer patients, good tumor pathologic response has been associated with a better outcome [15-18]. Whether this observation is due to a favorable action of the neo-adjuvant treatment or to biologically favorable tumor selection remains to be clarified. However, the emergence of new long-term oncological end-points such as tailoring treatment (omission of surgery for pCR patients) and sphincter conservation in low lying rectal cancer patients, makes the rate of pathological response as a new important early end-point [19]. While the limited number of patients included in the present trial precluded drawing any firm conclusions, the rates of $20 \%$ of pCR and $30 \%$ of major pPR are rather encouraging and in line with what has been reported in the other series using 5-FU with or without oxaliplatin $(10 \%-26 \%$ of pCR) $[1,20]$, or cetuximab + capecitabine (5\% - 25\% of pCR) [14]. However, this early end-point may vary widely due to tumor selection, interval between RT and surgery and the criteria used to define responses, and in the future uniform criteria are warranted to facilitate inter-comparison between studies.

While the series is too small to accurately evaluate the oncologic results, the 5-year actuarial loco-regional control rate was $84 \%$ and overall survival was $80 \%$, which is in line with what is reported recently in the literature [16-18]. The high rate of distant metastases $(30 \%)$ remains however of major concern, and the current approach requires improvement through the use of new drugs and/or new markers of tumor aggressiveness to identify patients with high risk of distant metastasis who may require intensive adjuvant or even neo-adjuvant systemic therapy.

\section{Conclusion}

Preoperative HFRT with the concomitant daily administration of $250 \mathrm{mg}$ of gefitinib was associated with promising early efficacy while associated with manageable acute toxicity. The major pathologic response rate is encouraging, although firm conclusions can't be drawn because of the limited number of patients included. For future studies caution should be made when combining multiple gastro-intestinal toxic agents concomitantly with RT and starting with lower doses of chemo- or biological agents is highly recommended. In the other hand distant metastasis still represents a concern and new strategies to overcome this issue are warranted. 


\section{Declaration of Interest}

The authors report no conflict of interest. The authors alone are responsible for the content and writing of the paper.

\section{Author's Contributions}

AA, AR and PG participated in the design of the study. $\mathrm{SB}, \mathrm{MB}$ and AFP performed the data management and AA the statistical analysis. SB and AA drafted the manuscript. All authors read and approved the final manuscript.

\section{REFERENCES}

[1] R. Sauer, H. Becker, W. Hohenberger, C. Rödel, C. Wittekind, R. Fietkau, et al., German Rectal Cancer Study Group, "Preoperative versus Postoperative Chemoradiotherapy for Rectal Cancer," The New England Journal of Medicine, Vol. 351, No.17, 2004, pp. 1731-1740. doi:10.1056/NEJMoa040694

[2] Swedish Rectal Cancer Trial, "Improved Survival with Preoperative Radiotherapy in Resectable Rectal Cancer," The New England Journal of Medicine, Vol. 336, No. 14, 1997, pp. 980-987. doi:10.1056/NEJM199704033361402

[3] C. Camma, M. Giunta, F. Fiorica, L. Pagliaro, A. Craxi and M. Cottone, "Preoperative Radiotherapy for Resectable Rectal Cancer: A Meta-Analysis," The Journal of American Medical Association, Vol. 284, No. 8, 2000, pp. 1008-1015. doi:10.1001/jama.284.8.1008

[4] E. Kapiteijn, C. A. Marijnen, I. D. Nagtegaal, H. Putter, W. H Steup, T. Wiggers, et al., Dutch Colorectal Cancer Group, "Preoperative Radiotherapy Combined with Total Mesorectal Excision for Resectable Rectal Cancer," The New England Journal of Medicine, Vol. 345, No. 9, 2001, pp. 638-646. doi:10.1056/NEJMoa010580

[5] B. G. Czito, C. G. Willett, J. C. Bendell, M. A. Morse, D. S. Tyler, N. H. Fernando, et al., "Increased Toxicity with Gefitinib, Capecitabine, and Radiation Therapy in Pancreatic and Rectal Cancer: Phase I Trial Results," Journal of Clinical Oncology, Vol. 24, No. 4, 2006, pp. 656-662. doi:10.1200/JCO.2005.04.1749

[6] V. Valentini, A. De Paoli, M. A. Gambacorta, G. Mantini, C. Ratto, F. M. Vecchio, et al., "Infusional 5-Fluorouracil and ZD1839 (Gefitinib-Iressa) in Combination with Preoperative Radiotherapy in Patients with Locally Advanced Rectal Cancer: A Phase I and II Trial (1839IL/0092)," International Journal of Radiation Oncology Biology Physics, Vol. 72, No. 3, 2008, pp. 644-649. doi:10.1016/j.ijrobp.2008.01.046

[7] A. S. Allal, S. Bieri, M. Bründler, C. Soravia, P. Gertsch, J. Bernier, et al., "Preoperative Hyperfractionated Radiotherapy for Locally Advanced Rectal Cancers: A Phase I-II Trial," International Journal of Radiation Oncology Biology Physics, Vol. 54, No. 4, 2002, pp. 1076-1081. doi:10.1016/S0360-3016(02)03003-1

[8] M. Baumann, M. Krause, E. Dikomey, K. Dittmann, W.
Dörr, U. Kasten-Pisula, et al., "EGFR-Targeted antiCancer Drugs in Radiotherapy: Preclinical Evaluation of Mechanisms," Radiotherapy Oncology, Vol. 83, No. 3, 2007, pp. 238-248. doi:10.1016/i.radonc.2007.04.006

[9] J. A. Bonner, P. M. Harari, J. Giralt, N. Azarnia, D. M. Shin, R. B. Cohen, et al., "Radiotherapy Plus Cetuximab for Squamous-Cell Carcinoma of the Head and Neck," The New England Journal of Medicine, Vol. 354, No. 6, 2006, pp. 567-578. doi:10.1056/NEJMoa053422

[10] K. J. Williams, B. A. Telfer, I. J. Stratford and S. R. Wedge, 'ZD1839 ('Iressa'), A Specific Oral Epidermal Growth Factor Receptor-Tyrosine Kinase Inhibitor, Potentiates Radiotherapy in a Human Colorectal Cancer Xenograft Mode," British Journal of Cancer, Vol. 86, No. 7, 2002, pp. 1157-1161. doi:10.1038/sj.bjc.6600182

[11] A. Trotti, R. Byhardt, J. Stetz, C. Gwede, B. Corn, K. Fu $\mathrm{K}$, et al., "Common Toxicity Criteria: Version 2.0. An Improved Reference for Grading the Acute Effects of cAncer Treatment: Impact on Radiotherapy," International Journal of Radiation Oncology Biology Physics, Vol. 47, No. 1, 2000, pp. 13-47. doi:10.1016/S0360-3016(99)00559-3

[12] B. G. Czito, J. C. Bendell, C. G. Willett, M. A. Morse, G. C. Blobe, D. S. Tyler, J. Thoma, et al., "Bevacizumab, Oxaliplatin, and Capecitabine with Radiation Therapy in Rectal Cancer: Phase I Trial Results," International Journal of Radiation Oncology Biology Physics, Vol. 68, No. 2, 2007, pp. 472-478. doi:10.1016/j.ijrobp.2007.02.001

[13] R. D. Hofheinz, K. Horisberger, C. Woernle, F. Wenz, U. Kraus-Tiefenbacher, G. Kähler, et al., "Phase I Trial of Cetuximab in Combination with Capecitabine, Weekly Irinotecan, and Radiotherapy as Neoadjuvant Therapy for Rectal Cancer," International Journal of Radiation Oncology Biology Physics, Vol. 66, No. 5, 2006, pp. 1384 1890. doi:10.1016/j.ijrobp.2006.07.005

[14] C. Rödel, D. Arnold, M. Hipp, T. Liersch, K. Dellas, I. Iesalnieks, et al., "Phase I-II Trial of Cetuximab, Capecitabine, Oxaliplatin, and Radiotherapy as Preoperative Treatment in Rectal Cancer," International Journal of Radiation Oncology Biology Physics, Vol. 70, No. 4, 2008, pp. 1081-1086. doi:10.1016/j.ijrobp.2007.07.2356

[15] M. Maas, P. J. Nelemans, V. Valentini, P. Das, C. Rödel, L. J. Kuo, et al., "Long-Term Outcome inPatients with a Pathological Complete Response afterChemoradiation for Rectal Cancer: A Pooled Analysisof Individual Patient Data," Lancet Oncology, Vol. 11, No. 9. 2010, pp. 835844. doi:10.1016/S1470-2045(10)70172-8

[16] C. Capirci, V. Valentini, L. Cionini, A. De Paoli, C. Rodel, R. Glynne-Jones, et al., "Prognostic Value of Pathologic Complete Response after Neoadjuvant Therapy in Locally Advanced Rectal Cancer: Long-Term Analysis of 566 ypCR Patients," International Journal of Radiation Oncology Biology Physics, Vol. 72, No. 1, 2008, pp. 99-107. doi:10.1016/j.ijrobp.2007.12.019

[17] C. Rödel, T. Liersch, R. M. Hermann, D. Arnold, T. Reese, M. Hipp, et al., "Multicenter Phase II Trial of Chemoradiation with Oxaliplatin for Rectal Cancer," Journal of Clinical Oncology, Vol. 25, No. 1, 2007, pp. 
110-117. doi:10.1200/JCO.2006.08.3675

[18] A. Rullier, C. Laurent, M. Capdepont, V. Vendrely, P. Bioulac-Sage and E. Rullier, "Impact of Tumor Response on Survival after Radiochemotherapy in Locally Advanced Rectal Carcinoma," American Journal of Surgical Pathology, Vol. 34, No. 4, 2010, pp. 562-568. doi:10.1097/PAS.0b013e3181d438b0

[19] A. S. Allal and C. Soravia, "Preoperative Radiotherapy for Rectal Cancer: Beyond Local Control," National Clinical Oncology, Vol. 3, No. 3, 2006, pp. 124-125. http://www.nature.com/nrclinonc/journal/v3/n3/full/ncpo nc0446.html

[20] D. I. Rosenthal, P. J. Catalano, D. G. Haller, J. C. Landry, E. R. Sigurdson, F. R. Spitz, et al., "Phase I Study of Preoperative Radiation Therapy with Concurrent Infusional 5-Fluorouracil and Oxaliplatin Followed by Surgery and Postoperative 5-Fluorouracil plus Leucovorin for T3/T4 Rectal Adenocarcinoma: ECOG E1297," International Journal of Radiation Oncology Biology Physics, Vol. 72, No. 1, 2008, pp. 108-113.

doi:10.1016/j.ijrobp.2008.05.054 